Arch. Math.

(C) 2021 The Author(s)

https://doi.org/10.1007/s00013-021-01637-6

Archiv der Mathematik

\title{
On the orthogonality of generalized eigenspaces for the Ornstein-Uhlenbeck operator
}

\author{
Valentina Casarino, Paolo Ciatti, and Peter Sjögren(i)
}

\begin{abstract}
We study the orthogonality of the generalized eigenspaces of an Ornstein-Uhlenbeck operator $\mathscr{L}$ in $\mathbb{R}^{N}$, with drift given by a real matrix $B$ whose eigenvalues have negative real parts. If $B$ has only one eigenvalue, we prove that any two distinct generalized eigenspaces of $\mathscr{L}$ are orthogonal with respect to the invariant Gaussian measure. Then we show by means of two examples that if $B$ admits distinct eigenvalues, the generalized eigenspaces of $\mathscr{L}$ may or may not be orthogonal.
\end{abstract}

Mathematics Subject Classification. 15A18, 47A70, 47D03.

Keywords. Ornstein-Uhlenbeck operator, Generalized eigenspaces, Orthogonality, Gaussian measure.

1. Introduction. In this note, we discuss the orthogonality of the generalized eigenspaces associated to a general Ornstein-Uhlenbeck operator $\mathscr{L}$ in $\mathbb{R}^{N}$.

Recently, the authors started studying some harmonic analysis issues in a nonsymmetric Gaussian context [1-3]. In particular, the Ornstein-Uhlenbeck semigroup $\left(\mathscr{H}_{t}\right)_{t>0}$ generated by $\mathscr{L}$ is not assumed to be self-adjoint in $L^{2}\left(\gamma_{\infty}\right)$; here $\gamma_{\infty}$ denotes the unique invariant probability measure under the action of the semigroup, and will be specified later.

In this general framework, the Ornstein-Uhlenbeck operator $\mathscr{L}$ admits a complete system of generalized eigenfunctions; see [8]. But without selfadjointness, the orthogonality of distinct eigenspaces of $\mathscr{L}$ is not guaranteed. In fact, while the kernel of $\mathscr{L}$ is always orthogonal to the other generalized eigenspaces of $\mathscr{L}$ in $L^{2}\left(\gamma_{\infty}\right)$, the question of orthogonality between generalized

The first and second authors are members of the Gruppo Nazionale per l'Analisi Matematica, la Probabilità e le loro Applicazioni (GNAMPA) of the Istituto Nazionale di Alta Matematica (INdAM) and were partially supported by GNAMPA (Project 2020 "Alla frontiera tra l'analisi complessa in più variabili e l'analisi armonica"). 
eigenspaces associated to nonzero eigenvalues is more delicate. As expected, the spectral properties of $B$ play a prominent role here. Indeed, we prove in Section 3 that if $B$ has a unique eigenvalue, then any two generalized eigenfunctions of $\mathscr{L}$ corresponding to different eigenvalues are orthogonal in $L^{2}\left(\gamma_{\infty}\right)$.

Then in Sections 4 and 5, we exhibit two examples showing, respectively, that if $B$ admits two distinct eigenvalues, the generalized eigenspaces associated to $\mathscr{L}$ may or may not be orthogonal. The last section also contains a result which relates the orthogonality of the eigenspaces of $\mathscr{L}$ to that of the eigenspaces of the drift matrix, under some restrictions.

In the following, the symbol $I_{k}$ will denote the identity matrix of size $k$, and we omit the subscript when the size is obvious. We will write $\langle\cdot, \cdot\rangle$ for scalar products both in $\mathbb{R}^{N}$ and in $L^{2}\left(\gamma_{\infty}\right)$. By $\mathbb{N}$ we mean $\{0,1, \ldots\}$.

2. The Ornstein-Uhlenbeck operator. In this section, we specify the definition of the Ornstein-Uhlenbeck operator $\mathscr{L}$ and recall some known facts concerning its spectrum.

We consider the Ornstein-Uhlenbeck semigroup $\left(\mathscr{H}_{t}^{Q, B}\right)_{t>0}$, given for all bounded continuous functions $f$ in $\mathbb{R}^{N}, N \geq 1$, and all $t>0$ by the Kolmogorov formula

$$
\mathscr{H}_{t}^{Q, B} f(x)=\int f\left(e^{t B} x-y\right) d \gamma_{t}(y), \quad x \in \mathbb{R}^{N},
$$

(see [6] and [7, Theorem 9.1.1]). Here $B$ is a real $N \times N$ matrix whose eigenvalues have negative real parts, and $Q$ is a real, symmetric, and positive-definite $N \times N$ matrix. Then we introduce the covariance matrices

$$
Q_{t}=\int_{0}^{t} e^{s B} Q e^{s B^{*}} d s, \quad t \in(0,+\infty],
$$

all symmetric and positive definite. Finally, the normalized Gaussian measures $\gamma_{t}$ are defined for $t \in(0,+\infty]$ by

$$
d \gamma_{t}(x)=(2 \pi)^{-\frac{N}{2}}\left(\operatorname{det} Q_{t}\right)^{-\frac{1}{2}} e^{-\frac{1}{2}\left\langle Q_{t}^{-1} x, x\right\rangle} d x .
$$

As mentioned above, $\gamma_{\infty}$ is the unique invariant probability measure of the Ornstein-Uhlenbeck semigroup.

The Ornstein-Uhlenbeck operator is the infinitesimal generator of the semigroup $\left(\mathscr{H}_{t}^{Q, B}\right)_{t>0}$, and it is explicitly given by

$$
\mathscr{L}^{Q, B} f(x)=\frac{1}{2} \operatorname{tr}\left(Q \nabla^{2} f\right)(x)+\langle B x, \nabla f(x)\rangle, \quad f \in \mathscr{S}\left(\mathbb{R}^{N}\right),
$$

where $\nabla$ is the gradient and $\nabla^{2}$ the Hessian.

By convention, we abbreviate $\mathscr{H}_{t}^{Q, B}$ and $\mathscr{L}^{Q, B}$ to $\mathscr{H}_{t}$ and $\mathscr{L}$, respectively. We can thus write $\mathscr{H}_{t}=e^{t \mathscr{L}}$.

In $[8$, Theorem 3.1], it is verified that the spectrum of $\mathscr{L}$ is the set

$$
\left\{\sum_{j=1}^{r} n_{j} \lambda_{j}: n_{j} \in \mathbb{N}\right\} \text {, }
$$


where $\lambda_{1}, \ldots, \lambda_{r}$ are the eigenvalues of the drift matrix $B$. In particular, 0 is an eigenvalue of $\mathscr{L}$, and the corresponding eigenspace ker $\mathscr{L}$ is one-dimensional and consists of all constant functions, as proved in [8, Section 3].

We also recall that, given a linear operator $T$ on some $L^{2}$ space, a number $\lambda \in \mathbb{C}$ is a generalized eigenvalue of $T$ if there exists a nonzero $u \in L^{2}$ such that $(T-\lambda I)^{k} u=0$ for some positive integer $k$. Then $u$ is called a generalized eigenfunction, and those $u$ span the generalized eigenspace corresponding to $\lambda$. As already recalled, it is known from [8, Section 3] that the OrnsteinUhlenbeck operator $\mathscr{L}$ admits a complete system of generalized eigenfunctions, that is, the linear span of the generalized eigenfunctions is dense in $L^{2}\left(\gamma_{\infty}\right)$. It is also known that all generalized eigenfunctions of $\mathscr{L}$ are polynomials, see [7, Theorem 9.3.20].

2.1. Use of Hermite polynomials. As proved in [9], a suitable linear change of coordinates in $\mathbb{R}^{N}$ makes $Q=I$ and $Q_{\infty}$ diagonal. When applying this, we adhere to the notation introduced in [4, Lemma 1], where also the following facts can be found. Let $\mathbf{H}_{n}$ denote the space of Hermite polynomials of degree $n$ in these coordinates, adapted by means of a dilation to $\gamma_{\infty}$ in the sense that the $\mathbf{H}_{n}$ are mutually orthogonal in $L^{2}\left(\gamma_{\infty}\right)$ (they are called $H_{\lambda, k}$ in [4]). The classical Hermite expansion (called the Itô-Wiener decomposition in [4]) says that $L^{2}\left(\gamma_{\infty}\right)$ is the closure of the direct sum of the $\mathbf{H}_{n}$; we refer to [10, p. 64] for a proof in dimension one and note that the extension to higher dimension is trivial. In other words, we can decompose any function $u \in L^{2}\left(\gamma_{\infty}\right)$ as

$$
u=\sum_{j} u_{j}
$$

with $u_{j} \in \mathbf{H}_{j}$ and convergence in $L^{2}\left(\gamma_{\infty}\right)$. Further, each $\mathbf{H}_{n}$ is invariant under $\mathscr{L}$; see [4, Proposition 1].

The Hermite decomposition implies, in particular, that each generalized eigenfunction of $\mathscr{L}$ with a nonzero eigenvalue is orthogonal to the space of constant functions, that is, to the kernel of $\mathscr{L}$. Anyway, we provide here a proof of this fact which is independent of Hermite polynomials.

Lemma 2.1. Let $\lambda \neq 0$. If $u \in L^{2}\left(\gamma_{\infty}\right)$ and $(\mathscr{L}-\lambda)^{k} u=0$ for some $k \in$ $\{1,2, \ldots\}$, then $\int u d \gamma_{\infty}=0$.

Proof. The implication is trivial if we set $k=0$, so assume it holds for some $k \geq 0$ and that $(\mathscr{L}-\lambda)^{k+1} u=0$.

Then

$$
\mathscr{L}(\mathscr{L}-\lambda)^{k} u=\lambda(\mathscr{L}-\lambda)^{k} u
$$

and thus for any $t>0$,

$$
e^{t \mathscr{L}}(\mathscr{L}-\lambda)^{k} u=e^{t \lambda}(\mathscr{L}-\lambda)^{k} u .
$$

These operators commute, so

$$
(\mathscr{L}-\lambda)^{k} e^{t \mathscr{L}} u=(\mathscr{L}-\lambda)^{k} e^{t \lambda} u,
$$

that is,

$$
(\mathscr{L}-\lambda)^{k}\left(e^{t \mathscr{L}} u-e^{t \lambda} u\right)=0
$$


The induction assumption now implies that

$$
\int\left(e^{t \mathscr{L}} u-e^{t \lambda} u\right) d \gamma_{\infty}=0 .
$$

Since $\gamma_{\infty}$ is invariant under the semigroup, this means that

$$
\int u d \gamma_{\infty}=e^{t \lambda} \int u d \gamma_{\infty}
$$

for all $t>0$. Thus the integral vanishes.

\section{The case when $B$ has only one eigenvalue.}

Proposition 3.1. If the drift matrix $B$ has only one eigenvalue, then any two generalized eigenfunctions of $\mathscr{L}$ with different eigenvalues are orthogonal with respect to $\gamma_{\infty}$.

Let $\lambda$ be the unique eigenvalue of $B$, which is necessarily real and negative. We first state a lemma and use it to prove the proposition. Recall that any generalized eigenfunction of $\mathscr{L}$ is a polynomial.

Lemma 3.2. Let $u$ be a generalized eigenfunction of $\mathscr{L}$ which is a polynomial of degree $n \geq 0$. Then the corresponding eigenvalue is $n \lambda$.

Proof of Proposition 3.1. Let $u$ be a generalized eigenfunction of $\mathscr{L}$, thus satisfying $(\mathscr{L}-\mu)^{k} u=0$ for some $\mu \in \mathbb{C}$ and $k \in \mathbb{N}$. Applying the coordinates from Subsection 2.1, we can decompose $u$ as in (2), where the sum is now finite. Since then

$$
\sum_{j}(\mathscr{L}-\mu)^{k} u_{j}=0
$$

and each term here is in the corresponding $\mathbf{H}_{j}$, all the terms are 0. But this is compatible with Lemma 3.2 only if there is only one nonzero term in the decomposition of $u$. Thus $u \in \mathbf{H}_{n}$, where $n$ is the polynomial degree of $u$.

Lemma 3.2 then implies that two generalized eigenfunctions with different eigenvalues are of different degrees and thus belong to different $\mathbf{H}_{n}$. The desired orthogonality now follows from that of the $\mathbf{H}_{n}$.

Proof of Lemma 3.2. Let $u$ be a generalized eigenfunction of $\mathscr{L}$ of polynomial degree $n$. We denote the corresponding eigenvalue by $\mu$. Decomposing $u$ as in (2), we see that this sum is for $j \leq n$ and that the term $u_{n}$ is nonzero and a generalized eigenfunction of $\mathscr{L}$ with eigenvalue $\mu$. For some $m$, the function $(\mathscr{L}-\mu)^{m} u_{n}$ will then be an eigenfunction with the same eigenvalue. This function is in $\mathbf{H}_{n}$ and thus a polynomial of degree $n$. As a result, we can assume that $u$ is actually an eigenfunction of $\mathscr{L}$ when proving the lemma.

We now choose coordinates in $\mathbb{R}^{N}$ that give a Jordan decomposition of $B$. This means that $B=\lambda I+R$, where $R=\left(R_{i, j}\right)$ is a matrix with nonzero entries only in the first subdiagonal. More precisely, $R_{i, i-1}=1$ for $i \in P$, where $P$ is a subset of $\{2, \ldots, N\}$, and all other entries of $R$ vanish.

We write $\mathscr{L}=\mathscr{S}+\mathscr{B}$, where

$$
\mathscr{B} f(x)=\langle B x, \nabla f(x)\rangle,
$$


and $\mathscr{S}$ is the remaining, second-degree part of $\mathscr{L}$. Notice that, when applied to polynomials, $\mathscr{B}$ preserves the degree whereas $\mathscr{S}$ decreases it by 2 . So if $v$ is the $n$ th-degree part of $u$, we must have $\mathscr{B} v=\mu v$.

We let $\mathscr{B}$ act on a monomial $x^{\alpha}$, where $\alpha \in \mathbb{N}^{N}$ is a multiindex of length $|\alpha|=n$, getting

$$
\begin{aligned}
\mathscr{B} x^{\alpha} & =\sum_{j} \lambda x_{j} \frac{\partial x^{\alpha}}{\partial x_{j}}+\sum_{i \in P} x_{i-1} \frac{\partial x^{\alpha}}{\partial x_{i}} \\
& =\lambda \sum_{j} \alpha_{j} x^{\alpha}+\sum_{i \in P} \alpha_{i} \frac{x_{i-1}}{x_{i}} x^{\alpha}=\lambda n x^{\alpha}+\sum_{i \in P} \alpha_{i} x^{\alpha^{(i)}},
\end{aligned}
$$

where $\alpha^{(i)}=\alpha+e_{i-1}-e_{i}$ for $i \in P$. Here $\left\{e_{j}\right\}_{j=1}^{n}$ denotes the standard basis in $\mathbb{R}^{N}$. Thus the restriction of $\mathscr{B}$ to the space of homogeneous polynomials of degree $n$ is given as $\lambda n I+\mathscr{R}$, where $\mathscr{R}$ is the linear operator that maps $x^{\alpha}$ to $\sum_{i \in P} \alpha_{i} x^{\alpha^{(i)}}$.

We claim that the only eigenvalue of $\mathscr{R}$ is 0 . If so, the only eigenvalue of the restriction of $\mathscr{B}$ mentioned above is $\lambda n$, which would prove the lemma since $\mathscr{B} v=\mu v$.

In order to prove this claim, we define for any $\alpha \in \mathbb{N}^{N}$ with $|\alpha|=n$,

$$
V(\alpha)=\sum_{1}^{N} j \alpha_{j}
$$

Clearly $V\left(\alpha^{(i)}\right)=V(\alpha)-1$. We select a basis in the linear space of all homogeneous polynomials of degree $n$ consisting of all monomials $x^{\alpha}$ with $|\alpha|=n$, enumerated in such a way that $V$ is nondecreasing. The definition of $\mathscr{R}$ now shows that its matrix with respect to this basis is upper triangular with zeros on the diagonal. The claim follows, and so does the lemma.

4. $\boldsymbol{B}$ has two distinct eigenvalues: a first example. The following example shows that the generalized eigenspaces of the Ornstein-Uhlenbeck operator may be orthogonal even in the case when $B$ has more than one eigenvalue. We show that $\mathscr{L}$, while not being self-adjoint, is normal; then the orthogonality of its eigenspaces follows from the spectral theorem.

In two dimensions, we let

$$
Q=I_{2} \quad \text { and } \quad B=\left(\begin{array}{cc}
-1 & 1 \\
-1 & -1
\end{array}\right)
$$

whose eigenvalues are $-1 \pm i$.

One finds that

and

$$
e^{s B}=e^{-s}\left(\begin{array}{cc}
\cos s & \sin s \\
-\sin s & \cos s
\end{array}\right)
$$

$$
e^{s B} e^{s B^{*}}=e^{-2 s} I_{2},
$$

so that

$$
Q_{\infty}=\frac{1}{2} I_{2}, \quad Q_{\infty}^{-1}=2 I_{2}
$$


We write

$$
B=-I_{2}+R, \quad \text { where } \quad R=\left(\begin{array}{cc}
0 & 1 \\
-1 & 0
\end{array}\right) .
$$

Since $R=-R^{*}$, [8, Proposition 2.1] implies that $\mathscr{L}$ is normal (observe that $I_{2}=\frac{1}{2} D_{1 / \lambda}$ in the notation of [8]).

However, we give below a brief, direct proof of this fact, independent of the change of variables adopted in $[8,9]$. In the following, we write

$$
\mathscr{L}=\mathscr{L}^{0}+\mathscr{R},
$$

where

$$
\mathscr{L}^{0}=\frac{1}{2} \Delta-\langle x, \nabla\rangle
$$

is the standard Ornstein-Uhlenbeck operator and so self-adjoint in $L^{2}\left(\gamma_{\infty}\right)$. Further,

$$
\mathscr{R}=\langle R x, \nabla\rangle
$$

is seen to be an antisymmetric operator in $L^{2}\left(\gamma_{\infty}\right)$. This leads to

$$
\left[\mathscr{L}, \mathscr{L}^{*}\right]=\left[\mathscr{L}^{0}+\mathscr{R}, \mathscr{L}^{0}-\mathscr{R}\right]=-\mathscr{L}^{0} \mathscr{R}+\mathscr{R} \mathscr{L}^{0}-\mathscr{L}^{0} \mathscr{R}+\mathscr{R} \mathscr{L}^{0}=2\left[\mathscr{R}, \mathscr{L}^{0}\right] .
$$

If we write $\partial_{i}$ for $\partial_{x_{i}}, i=1,2$, this amounts to

$$
2\left[x_{2} \partial_{1}-x_{1} \partial_{2}, \frac{1}{2} \Delta-x_{1} \partial_{1}-x_{2} \partial_{2}\right] .
$$

A straightforward computation shows that this vanishes, and so $\mathscr{L}$ is normal. The spectral theorem for normal operators now implies the following result.

Proposition 4.1. With $N=2$, let $Q$ and $B$ be as in (3). Then each generalized eigenfunction of $\mathscr{L}$ is an eigenfunction. Moreover, any two eigenfunctions of $\mathscr{L}$ with different eigenvalues are orthogonal with respect to $\gamma_{\infty}$.

5. $\boldsymbol{B}$ has two distinct eigenvalues: a second example. In this section, we exhibit a class of drift matrices $B$ with two different eigenvalues (which, in contrast to those in the example in Section 4, are real), but such that the generalized eigenspaces associated to the corresponding Ornstein-Uhlenbeck operator $\mathscr{L}$ are not orthogonal.

In $\mathbb{R}^{2}$, we consider $Q=I_{2}$ and

$$
B=\left(\begin{array}{cc}
-a+d & 0 \\
c & -a-d
\end{array}\right),
$$

with $a>d>0$ and $c \neq 0$. To compute the exponential of $s B$, we write $B=-a I+M$, where

$$
M=\left(\begin{array}{cc}
d & 0 \\
c & -d
\end{array}\right) .
$$

Since $M M=d^{2} I$, we get for $s>0$,

$$
\exp (s B)=e^{-a s}\left(\cosh (s d) I+d^{-1} \sinh (s d) M\right) \text {. }
$$


This leads to

$$
\exp (s B) \exp \left(s B^{*}\right)=e^{-2 a s}\left(\begin{array}{cc}
e^{2 s d} & \frac{c}{d} e^{s d} \sinh (s d) \\
\frac{c}{d} e^{s d} \sinh (s d) & \frac{c^{2}}{d^{2}} \sinh ^{2}(s d)+e^{-2 s d}
\end{array}\right) .
$$

Integrating this matrix over $0<s<\infty$, we obtain

$$
Q_{\infty}=\left(\begin{array}{cc}
\frac{1}{2(a-d)} & \frac{c}{4 a(a-d)} \\
\frac{c}{4 a(a-d)} & \frac{c^{2}}{4 a(a-d)(a+d)}+\frac{1}{2(a+d)}
\end{array}\right),
$$

and so

$$
\frac{1}{2} Q_{\infty}^{-1}=\frac{1}{c^{2}+4 a^{2}}\left(\begin{array}{cc}
2 a\left[c^{2}+2 a(a-d)\right] & -2 a c(a+d) \\
-2 a c(a+d) & 4 a^{2}(a+d)
\end{array}\right) .
$$

The invariant measure $\gamma_{\infty}$ is thus proportional to

$$
\begin{aligned}
& \exp \left(-\frac{2 a\left[c^{2}+2 a(a-d)\right]}{c^{2}+4 a^{2}} x_{1}^{2}+\frac{4 a c(a+d)}{c^{2}+4 a^{2}} x_{1} x_{2}-\frac{4 a^{2}(a+d)}{c^{2}+4 a^{2}} x_{2}^{2}\right) d x \\
& \quad=\exp \left(-(a-d) x_{1}^{2}\right) \exp \left(-\frac{a+d}{c^{2}+4 a^{2}}\left(c x_{1}-2 a x_{2}\right)^{2}\right) d x
\end{aligned}
$$

Writing $z_{1}=\sqrt{a-d} x_{1}$ and $z_{2}=\sqrt{\frac{a+d}{c^{2}+4 a^{2}}}\left(2 a x_{2}-c x_{1}\right)$ and recalling that $\gamma_{\infty}$ is a probability measure, we see that

$$
d \gamma_{\infty}=\pi^{-1} \exp \left(-z_{1}^{2}-z_{2}^{2}\right) d z .
$$

To find some eigenfunctions of $\mathscr{L}$, we consider polynomials in $x_{1}, x_{2}$ of degree 2. One finds that

$$
\begin{aligned}
& v_{1}=x_{1}^{2}-\frac{1}{2(a-d)}, \\
& v_{2}=x_{1}^{2}-\frac{2 d}{c} x_{1} x_{2}-\frac{1}{2 a}, \\
& v_{3}=x_{1}^{2}-\frac{4 d}{c} x_{1} x_{2}+\frac{4 d^{2}}{c^{2}} x_{2}^{2}-\frac{c^{2}+4 d^{2}}{2 c^{2}(a+d)}
\end{aligned}
$$

are eigenfunctions, with eigenvalues $-2(a-d),-2 a$, and $-2(a+d)$, respectively.

Any two of these polynomials turn out not to be orthogonal with respect to the invariant measure, as follows by straightforward computations. We sketch one example.

One simply multiplies $v_{1}$ and $v_{3}$ and rewrites the product in terms of $z_{1}$ and $z_{2}$. Doing so, one can neglect all terms of odd order in $z_{1}$ or $z_{3}$, when integrating with respect to $\gamma_{\infty}$. Writing "odd" for such terms, we find that the product is

$$
\begin{aligned}
& \frac{1}{a^{2}} z_{1}^{4}+\frac{d^{2}\left(c^{2}+4 a^{2}\right)}{a^{2} c^{2}\left(a^{2}-d^{2}\right)} z_{1}^{2} z_{2}^{2}-\left[\frac{c^{2}+4 d^{2}}{2 c^{2}\left(a^{2}-d^{2}\right)}+\frac{1}{2 a^{2}}\right] z_{1}^{2} \\
& -\frac{d^{2}\left(c^{2}+4 a^{2}\right)}{2 a^{2} c^{2}\left(a^{2}-d^{2}\right)} z_{2}^{2}+\frac{c^{2}+4 d^{2}}{4 c^{2}\left(a^{2}-d^{2}\right)}+\text { odd. }
\end{aligned}
$$


Integrating and simplifying, we get

$$
\int v_{1} v_{3} d \gamma_{\infty}=\frac{1}{2 a^{2}}>0
$$

so $v_{1}$ and $v_{3}$ are not orthogonal.

Remark 5.1. Let now $d=a / 2$ in this example. Then the fourth-degree polynomial

$$
v_{4}=x_{1}^{4}-\frac{6}{a} x_{1}^{2}+\frac{3}{a^{2}}
$$

is an eigenfunction of $\mathscr{L}$ with eigenvalue $-2 a$, like $v_{2}$. Thus eigenfunctions of different polynomial degrees can have the same eigenvalue. This shows that for an eigenfunction $u$, the sum in (2) may consist of more than one term, and a (generalized) eigenspace need not be contained in one $\mathbf{H}_{n}$.

The eigenvalues of the matrix $B$ defined in (4) are $-a \pm d$, and it is easily seen that the corresponding eigenspaces are not orthogonal in $\mathbb{R}^{2}$. This turns out to be related to the non-orthogonality of the eigenspaces of $\mathscr{L}$, at least in two dimensions, in the following way.

Proposition 5.2. Let $N=2$ and $Q=I$, and assume that $B$ has two different, real eigenvalues. Then the generalized eigenspaces of $\mathscr{L}$ are orthogonal in $L^{2}\left(\gamma_{\infty}\right)$ if and only if the two eigenspaces of $B$ are orthogonal in $\mathbb{R}^{2}$.

Proof. To begin with, we consider a coordinate change $\widetilde{x}=H x$, where $H$ is an orthogonal matrix. Simple computations show that the operator $\mathscr{L}^{Q, B}$ is transformed to $\mathscr{L}^{\widetilde{Q}, \widetilde{B}}$ in the new coordinates, with $\widetilde{Q}=H Q H^{*}$ and $\widetilde{B}=$ $H B H^{*}$; cf. [9, p. 474]. In our case, $\widetilde{Q}=Q=I$. The eigenvalues of $B$ and the angle between its eigenvectors will not change.

To prove the proposition, assume first that the (real) eigenvectors of $B$ are orthogonal in $\mathbb{R}^{2}$. Then $B$ is symmetric since it can be diagonalized by means of an orthogonal change of coordinates as just described. This implies that $\mathscr{L}$ is symmetric ([7, Proposition 9.3 .10$])$, so that the orthogonality of its eigenspaces is trivial.

Next, we assume that the eigenvectors of $B$ are not orthogonal in $\mathbb{R}^{2}$. By Schur's decomposition theorem (see [5, Theorem 2.3.1]), there exists an orthogonal change of coordinates which makes $B$ lower triangular, though not diagonal. We are thus in the situation described in (4). As we have seen, some eigenspaces of $\mathscr{L}$ are then not orthogonal with respect to the invariant measure.

We finally remark that the "if" part of this proposition easily extends to arbitrary dimension $N$. Then it is assumed that $B$ has $N$ different, real eigenvalues with mutually orthogonal eigenspaces. 
Funding Open access funding provided by University of Gothenburg.

Open Access. This article is licensed under a Creative Commons Attribution 4.0 International License, which permits use, sharing, adaptation, distribution and reproduction in any medium or format, as long as you give appropriate credit to the original author(s) and the source, provide a link to the Creative Commons licence, and indicate if changes were made. The images or other third party material in this article are included in the article's Creative Commons licence, unless indicated otherwise in a credit line to the material. If material is not included in the article's Creative Commons licence and your intended use is not permitted by statutory regulation or exceeds the permitted use, you will need to obtain permission directly from the copyright holder. To view a copy of this licence, visit http://creativecommons. org/licenses/by/4.0/.

Publisher's Note Springer Nature remains neutral with regard to jurisdictional claims in published maps and institutional affiliations.

\section{References}

[1] Casarino, V., Ciatti, P., Sjögren, P.: The maximal operator of a normal OrnsteinUhlenbeck semigroup is of weak type $(1,1)$. Ann. Sc. Norm. Sup. Pisa Cl. Sci. (5) XXI, 385-410 (2020)

[2] Casarino, V., Ciatti, P., Sjögren, P.: On the maximal operator of a general Ornstein-Uhlenbeck semigroup. arXiv:1901.04823 (2020)

[3] Casarino, V., Ciatti, P., Sjögren, P.: Riesz transforms of a general OrnsteinUhlenbeck semigroup. Calc. Var. Partial Differential Equations 60(4), 135 (2021)

[4] Chill, R., Fasangova, E., Metafune, G., Pallara, D.: The sector of analyticity of the Ornstein-Uhlenbeck semigroup on $L^{p}$ spaces with respect to invariant measure. J. Lond. Math. Soc. (2) 71, 703-722 (2005)

[5] Horn, R.A., Johnson, C.R.: Matrix Analysis. Cambridge University Press, Cambridge (2012)

[6] Kolmogorov, A.N.: Zufällige Bewegungen. Ann. Math. (2) 35(1), 116-117 (1934)

[7] Lorenzi, L., Bertoldi, M.: Analytical Methods for Markov Semigroups. Pure and Applied Mathematics (Boca Raton), vol. 283. Chapman \& Hall/CRC, Boca Raton (2007)

[8] Metafune, G., Pallara, D., Priola, E.: Spectrum of Ornstein-Uhlenbeck operators in $L^{p}$ spaces with respect to invariant measures. J. Funct. Anal. 196, 40-60 (2002)

[9] Metafune, G., Prüss, J., Rhandi, A., Schnaubelt, R.: The domain of the Ornstein-Uhlenbeck operator on a $L^{p}$-space with invariant measure. Ann. Sc. Norm. Super. Pisa Cl. Sci. 1, 471-487 (2002)

[10] Wiener, N.: The Fourier Integral and Certain of its Applications. Cambridge University Press, Cambridge (1933) 
Valentina Casarino

Università degli Studi di Padova

Stradella san Nicola 3

36100 Vicenza

Italy

e-mail: valentina.casarino@unipd.it

Paolo Ciatti

Università degli Studi di Padova

Via Marzolo 9

35100 Padua

Italy

e-mail: paolo.ciatti@unipd.it

PETER SJÖGREN

Department of Mathematical Sciences

University of Gothenburg and

Chalmers University of Technology

SE-41296 Göteborg

Sweden

e-mail: peters@chalmers.se

Received: 18 March 2021

Revised: 4 June 2021

Accepted: 20 June 2021. 